\title{
Continuous Wave MRI of Heterogeneous Materials
}

Andrew J. Fagan*, Gareth R. Davies, James M.S. Hutchison, David J. Lurie

Department of Bio-Medical Physics and Bio-Engineering,

University of Aberdeen,

Foresterhill,

Aberdeen AB25 2ZD,

U.K.

*To whom correspondence should be addressed

Telephone number: +44 1224 552861; fax number: +44 1224685645

e-mail a.fagan@biomed.abdn.ac.uk 


\begin{abstract}
A prototype continuous wave MRI system operating at $7 \mathrm{~T}$ has been used successfully to study a variety of heterogeneous materials exhibiting $T_{2}$ relaxation values ranging from $10 \mu$ s to 50 ms. Two-dimensional images of a poly(methyl methacrylate) (PMMA) resolution phantom $\left(\mathrm{T}_{2}=38 \mu \mathrm{s}\right)$ exhibited a spatial resolution of approximately $1 \mathrm{~mm}$ at a magnetic field gradient strength of $200 \mathrm{mT} / \mathrm{m}$. The technique was used to study the hydration, drying and subsequent water penetration properties of cement samples made from ordinary Portland cement, and revealed inhomogeneities arising from the cure conditions. Sandstone samples from an oil reservoir in the North Sea were also studied; structure within these materials, arising from the sedimentary bed layering in the reservoir, was found to have an effect on their water transport properties. A section from a confectionery bar $\left(\mathrm{T}_{2}{ }^{*}\right.$ approximately 50-60 ms) was also imaged, and its internal structure could be clearly discerned.
\end{abstract}

\title{
Keywords:
}

Continuous-Wave, Solid-imaging, Short- $\mathrm{T}_{2}$, Heterogeneous, MRI 


\section{Introduction}

The difficulties associated with imaging materials in the solid state have been well documented [1-4]. Strong relaxation effects due to dipolar interactions result in very short $\mathrm{T}_{2}$ relaxation times, often much shorter than the dead-time of techniques employing conventional spin-echo methods. A number of approaches have been developed to overcome these problems, the most successful of which involve the use of large gradients to overcome the broad linewidths [5,6], or use pure phase-encoded acquisition with modest gradient strengths [7]. Techniques employing very strong magnetic field gradients include: stray field imaging (STRAFI), which uses the very large static magnetic field gradient present in the fringe field of a superconducting magnet, together with repeated acquisitions between which the sample is moved or the magnetic field or RF frequency is adjusted, to determine the spin density of successive slices through the sample $[5,8]$; and the oscillating gradient technique, which incorporates the gradient coils within a resonant circuit, so that modest gradient strengths can be obtained relatively easily $[6,9,10]$. The single point imaging technique (SPI, or its variant SPRITE, single-point ramped imaging with $\mathrm{T}_{1}$ enhancement) is a pure phase-encoding method, which acquires a single data sample at a fixed time after the application of an RF pulse, and repeats this as the gradient is incremented [7,11,12].

Heterogeneous materials such as porous media are commonplace throughout nature, and are becoming increasingly prevalent in industry and technology. There is a growing interest in the ability to non-invasively study such materials, whether it be to gain an understanding of the transport properties of fluids in cementitious materials [13,14], or to visualise the micro-structure of trabecular bone [15]. However, the application of MR techniques to the study of porous media poses additional challenges: the presence of paramagnetic impurities, susceptibility-induced field gradients and chemical shift anisotropies all result in enhanced relaxation and, hence, broader resonance lines. Furthermore, the presence of liquids in such confined geometries can lead to adsorption phenomena which often exhibit multi-exponential signal decay [16,17], and thus techniques developed to study these materials must be capable of spanning a broad range of $\mathrm{T}_{2}$ values (from $\mu$ s to ms). 
Solid imaging techniques have been used to study porous media, although no one technique has emerged as an all-round gold standard. Technical limitations on the oscillation period $\left(\mathrm{TE}_{\min } \approx 200 \mu \mathrm{s}\right.$ ) limit the shortest measurable relaxation time amenable to the oscillating gradient method $[9,18]$. STRAFI, an inherently 1-dimensional technique (although multidimensional imaging has been reported recently [19]), has been used to study porous media with a broad range of $T_{2}$ values $[14,20,21]$, although it requires the use of specialised hardware and its heavily relaxation-weighted signal can be difficult to interpret [22]. Perhaps the most successful approach to date has used SPI/SPRITE techniques, with images of porous materials with $\mathrm{T}_{2}{ }^{*}$ values as low as $50 \mu$ s reported in the literature [23-25].

The technique described in this paper utilises continuous-wave detection, originally developed for electron paramagnetic resonance (EPR) experiments but adapted here to the study of materials in the solid state by NMR. In EPR, materials with relaxation times less than $1 \mu$ s are typically encountered; the signal from the sample under investigation is detected by recording the change in the electrical characteristics of the RF resonator as the sample is swept through resonance by a time-varying magnetic field applied across the sample. The use of a continuous wave of RF energy eliminates the dead time inherent in all other techniques, thus effectively removing the limit on the lowest value of $\mathrm{T}_{2}$ that can be investigated. Furthermore, the problems associated with rapid gradient switching are avoided through the use of continuously-applied gradients. Unlike pulsed solid imaging methods, however, extracting relaxation-time contrast information from samples is not so straightforward, although strategies to address this problem have been discussed previously [26]. As with other techniques, the achievable spatial resolution is ultimately limited by the applied magnetic field gradient strength. A prototype system incorporating these features has been described in an earlier paper [26]. In the present work, we describe on-going developments to this system and demonstrate the versatility of the technique by investigating several heterogeneous material systems. 


\section{Experimental}

Figure 1 shows a block diagram of the CW-MRI apparatus, based on a 7 T, $183 \mathrm{~mm}$ diameter horizontal bore superconducting magnet (Oxford Instruments, UK). The home-built water-cooled coil assembly consists of a Golay X-gradient coil set (250 mT/m maximum gradient), a Maxwell pair to generate the $\mathrm{Z}$ gradient (250 mT/m maximum gradient), and a split solenoid coil to provide magnetic field sweep (up to $\pm 7 \mathrm{mT}$ ). We use an eight-leg, high-pass birdcage resonator with diameter $52 \mathrm{~mm}$ and length $80 \mathrm{~mm}$, tuned to $300 \mathrm{MHz}(\mathrm{Q}=300$ when matched to $50 \Omega)$. The resonator was built to be as free as possible from hydrogen protons, which otherwise would be picked up as background signals. To this end, the resonator was supported by PTFE end rings, and the fixed tuning capacitors used were miniature chip capacitors (Tekelec, France). A brass RF shield with i/d 73 mm, o/d 76 mm, and length $204 \mathrm{~mm}$, fitted inside the coil assembly. The sample was loaded into the resonator using a ${ }^{1} \mathrm{H}$-free manipulator, consisting of a PTFE sample holder supported by a glass rod. Tuning and coupling of the resonator was done using a network analyser (Hewlett Packard, USA, Model 8712ET).

At the heart of the system lies the continuous-wave spectrometer, illustrated in the dashed box in Figure 1. A synthesiser (Hewlett Packard, USA, Model HP8647A) was used to apply RF at one specific frequency to one terminal of the home-built quadrature-hybrid junction. The CW-MRI technique works as follows: Upon loading the sample, the resonator is tuned and matched to $50 \Omega$, at which point the RF power is equally split between the resonator and the $50 \Omega$ load. The magnetic field applied across the sample is then swept using a time-varying ramped magnetic field, and at resonance the spins absorb energy, which changes the impedance of the resonator, leading to an impedance mismatch across the hybrid junction. This mismatch causes power to be transmitted through the junction to the detector, and a plot of detector output versus magnetic field shows the sample’s magnetic resonance absorption spectrum. The change in signal as resonance is achieved is often small compared to the electrical noise present in the system, and therefore an audiofrequency modulation is superimposed on the ramped magnetic field, with lock-in detection used to extract the signal with the same frequency as the modulation and at a fixed relative phase. 
The output from the lock-in amplifier (Stanford Research Systems, Model 830 DSP) is proportional to the change in signal reflected from the resonator as the field is swept, i.e. it is the first derivative of the signal. The RF shield was split along its length to prevent the magnetic field modulation causing eddy currents, which would introduce acoustic interference and prevent the modulation from penetrating the shield to the sample. The split was closed to RF penetration by forming a capacitor comprising the shield itself and a strip of copper (20 mm wide, $40 \mu \mathrm{m}$ thick) bridging the gap, with a strip of PTFE in between acting as the dielectric [26].

The application of a magnetic field gradient creates a plane of resonance perpendicular to the gradient direction; the effect of the ramped magnetic field is then to sweep this plane of resonance across the sample. In this way, a one-dimensional line profile is acquired, with each point in the profile representing the first derivative of the spin density of the sample projected onto the axis of the gradient. Rotating the gradient direction in a fixed plane around the sample results in a series of projections, from which a two-dimensional image can be reconstructed using filtered backprojection. Extension to three dimensions is straightforward, although only 1- and 2dimensional work is described here.

The system is controlled by an $800 \mathrm{MHz}$ Pentium III PC, with the sequence control and data acquisition software written in LabVIEW ${ }^{\mathrm{TM}}$ (National Instruments, USA). The waveform for the ramped magnetic field is generated in the PC and output via a multifunction I/O board (National Instruments, USA, Model PCI 4060E) to the homebuilt solenoid power supply, where it is combined with the modulation signal ( $\sim 1 \mathrm{kHz}$ ) from the lock-in amplifier. The diode detector converts the amplitudemodulated RF signal to a signal at the modulation frequency. After amplification, this signal is split between the lock-in amplifier and a home-built automatic frequency controller (AFC), which compensates for drifts of up to $\pm 50 \mathrm{kHz}$ in the resonator's tuning by adjusting the RF source frequency. It is necessary to match all such frequency drifts with a corresponding magnetic field offset, which is accomplished by modifying the magnetic field ramp. To do this, an output from the AFC, proportional to the instantaneous frequency offset value, is sent to the PC, where it is digitised and dynamically added to the ramp pattern. Each step of the ramp thus modified is then 
placed in a data buffer before being output to the solenoid power supply in a timed fashion. The RF source, lock-in amplifier and gradient power supplies (Advance Hivolt, UK, Model AP6050) are controlled through an IEEE 488 GPIB bus. Data processing is carried out using LabVIEW ${ }^{\mathrm{TM}}$, while image reconstruction is carried out using Interactive Data Language (Research Systems Inc., USA).

\section{Acquisition Parameters}

One-dimensional line profiling along the axis of cylindrically-shaped samples and 2dimensional imaging of samples with 2-D geometries were carried out. For 1-D profiling experiments, a field sweep time of 4 seconds in conjunction with 40 signal averages to improve the signal/noise ratio was used consistently, giving a total acquisition time of approximately 4.5 minutes. Thus processes with timescales of the order of minutes (for example, water penetration into rocks and cements) could be studied with good signal/noise characteristics. For 2-D imaging experiments, field sweep times ranging from 4 to 8 seconds with 2 - 4 signal averages were typically used, together with the acquisition of 32 - 64 projections around the sample, resulting in acquisition times in the range $10-20$ minutes. The RF power used throughout was in the $\mathrm{mW}$ range, generally dependent on the $\mathrm{T}_{1}$ of the material under investigation (low powers must be used, as it is easy to saturate the spin system with continuous RF irradiation). The amplitude of the magnetic field modulation used depended on the sample's $T_{2}$, with a smaller $T_{2}$ value (i.e. broader linewidth) permitting the use of a larger modulation amplitude. The use of a larger modulation leads to a larger SNR, but at the expense of introducing line broadening; the optimal situation is to use a modulation equivalent to half the linewidth of the material under study (typically in the range of 80 to $800 \mu \mathrm{T}$ with the present experimental configuration).

\section{Sample Preparation}

(i) Cement-1D Experiments: A range of cement samples was fabricated using

Ordinary Portland Cement with a variety of water/cement ratios, cast in cylindrical moulds measuring $30 \mathrm{~mm}$ in diameter and $30 \mathrm{~mm}$ in depth, and cured in a variety of environments. We present here the results from one particular sample, with a water/cement ratio of 0.4 by weight (which is close to optimal for this material), which was cured for 28 days in a dry environment (relative humidity $<5 \%$ ). All 
surfaces of the sample except the top face were sealed with Parafilm, to promote evaporation of water through this surface only. This was done to disrupt the hydration process in this part of the sample and hence introduce some inhomogeneity into the cured sample. Following the cure period, the sealing was removed and the sample was dried in an oven for 48 hours at $110^{\circ} \mathrm{C}$ to remove all evaporable water. (ii) Cement-2D Experiments: For 2-D imaging experiments, a sample with a rectangular cross-section was prepared (dimensions 30 × 32 x 12 mm) using Ordinary Portland Cement with a water/cement ratio of 0.4. The sample was moist-cured in a wet environment (relative humidity $>95 \%$ ) for 28 days to ensure a homogeneous cure, and dried at $110^{\circ} \mathrm{C}$ for 48 hours to remove all evaporable water. The sample was then completely sealed except for the left face, which was exposed to a water reservoir. The water reservoir was removed for imaging, and an acquisition time of 15 minutes was used for each image (there was negligible penetration of water into the sample while the water reservoir was removed).

(iii) Rock Core Samples: 1D profiling experiments were carried out on two cylindrical North Sea oil reservoir sandstone samples. The core samples, which had dimensions $40 \mathrm{~mm}$ long and $25 \mathrm{~mm}$ in diameter, were cut in two orientations: with their axis either parallel or perpendicular to the sedimentary layers in the reservoir sea bed. In each case, the samples were sealed except for the left face, which was placed in contact with a water reservoir (removed for profiling).

\section{Results and Discussion}

\section{PMMA Resolution Phantom}

Figure 2 shows the image of a poly(methyl methacrylate) (PMMA) (Perspex / Plexiglas) resolution phantom with a $\mathrm{T}_{2}$ of $38 \mu$ s [27], acquired using a gradient of $200 \mathrm{mT} / \mathrm{m}$ (acquisition time $=30$ minutes). The unprocessed image is displayed in Figure 2(b), while Figure 2(c) displays the effect of deconvolution of this image with its zero gradient spectrum using a modified Wiener filter algorithm. This deconvolution was possible since the spectral linewidth did not vary with position within this sample, and results in a marked improvement in overall image quality. The spatial resolution in CW-MRI is given by: 


$$
\Delta \mathrm{x}=2 \pi \Delta \mathrm{f} /(\gamma \mathrm{G})
$$

where $\Delta \mathrm{f}$ is the natural linewidth, $\gamma$ is the gyromagnetic ratio, and $\mathrm{G}$ is the applied magnetic field gradient. However, $\Delta \mathrm{f}$ can be approximated by:

$$
\Delta \mathrm{f} \cong 1 /\left(\pi \mathrm{T}_{2}\right)
$$

and hence we have:

$$
\Delta \mathrm{x}=2 /\left(\gamma \mathrm{GT}_{2}\right)
$$

The smallest hole in the phantom ( $2 \mathrm{~mm}$ ) is well resolved, indicating that we are close to achieving the theoretical maximum spatial resolution of approximately $1 \mathrm{~mm}$ for this material at this gradient strength. The increase in signal intensity evident at the top and bottom of the phantom is due to its extension into areas of RF non-uniformity close to the resonator's walls.

\section{Cementitious Materials - 1-Dimensional Experiments}

One-dimensional profiling experiments carried out on the cylindrical cement sample during curing and drying are illustrated in Figure 3. The peak on the left is a reference material made from an ABS polymer $(1 \mathrm{~mm}$ wide, containing two distinct linewidth-components), which was used to scale intensity variations due to drifting of the instrumentation during the course of the experiment (generally less than $2 \%$ ). The profiles displayed in Figure 3 have been normalised with respect to the intensity of the reference material. It can be seen that there is a gradual decrease in intensity on the left-hand side of the profile, consistent with a loss of water from this part of the sample through evaporation from the exposed face. It should be noted that our technique detects water protons in all environments within the sample, that is capillary water, gel water and chemically-combined water. Consequently, any decrease in the measured intensity results from a loss of water from the sample, rather than a loss of signal due to an increasing proportion of chemically-combined water as the hydration progresses. The maximum non-uniformity measured along the sample occurred after 14 days of curing, after which the profile began to level off as water diffused from the right side of the sample and was eventually lost through evaporation. There was no further decrease in intensity or indeed sample mass after 24 hours of drying, and hence the 24-hour profile is a measure of the chemically combined water content within the sample. Note the residual slope to the left hand side in this profile, indicating an inhomogeneous cure along the axis of the sample. 
This inhomogeneity was further revealed by water penetration studies, which demonstrated an enhanced uptake of water in the left side, consistent with a more porous structure (i.e. incomplete hydration) in this part of the sample (data not shown).

Measurements of the linewidth of the sample as a function of curing and drying times are illustrated in Figure 4. These measurements were made with no applied field gradient, with the linewidth calculated from the full-width-half-maximum of the measured curves. A small $(80 \mu \mathrm{T})$ magnetic field modulation was used in order to minimise any broadening of the linewidths caused by the modulation itself. It is likely that the linewidth comprises contributions deriving from ${ }^{1} \mathrm{H}$ protons in a range of different $\mathrm{T}_{2}$ environments; however, no extraction of individual linewidths was performed, rather the overall aggregated linewidth was measured in each case. Nevertheless, one can discern a gradual increase in linewidth, reaching a maximum of $\sim 33 \mathrm{kHz}$ after 24 hours of drying, which corresponds to a $\mathrm{T}_{2}{ }^{*}$ of $\sim 10 \mu \mathrm{s}$.

\section{Cementitious Materials - 2-Dimensional Experiments}

Figure 5 shows the results of water penetration into the rectangular cement sample. It can be seen that the water has almost fully penetrated across the sample after approximately 1 hour of soaking, with a further increase in intensity after prolonged soaking, consistent with the filling of smaller pores within the sample. Note that the signal in the right part of figure 5(a) derives solely from the chemically combined water in the sample, with a $\mathrm{T}_{2}{ }^{*}$ of approximately $10 \mu \mathrm{s}$.

\section{Sandstone Rocks}

There is considerable interest within the oil industry in characterising the transport properties of liquids in reservoir rock in order to optimise oil extraction. Indeed, petrophysical applications of NMR abound, including imaging irreducible water in cored reservoir samples [18], measurements of pore size distributions and wettability in chalk samples [28], and fluid flow characterisation in oil reservoir rocks [29,30]. Naturally occurring deformations within rocks and layering effects in sea beds can 
have dramatic effects on fluid flow, and can ultimately affect the overall recoverable resource in a reservoir [31].

Figure 6(a) shows the results of the water penetration into the 'parallel' sandstone sample: one can see that after 8 hours of soaking, the sample is almost completely soaked. However, there is considerably less penetration into the 'perpendicular' sample after 8 hours of soaking, as illustrated in Figure 6(b), as would be expected since the water penetrating this sample must traverse the boundaries between layers, rather than travel along channels formed by the layers in the 'parallel' sample. Indeed, this layering effect in the 'perpendicular' sample is evident as ripples in the 8-, 20- and 24-hour profiles.

\section{Chocolate Sample}

An ideal imaging technique should be capable of spanning a wide range of $T_{2}$ values. To demonstrate the versatility of the CW-MRI technique, a sample from a common chocolate bar (Snickers ${ }^{\mathrm{TM}}$ ) was prepared and imaged. The sample, which was taken from the top section of the bar, contained peanuts in a caramel matrix and measured $42 \times 25 \times 4 \mathrm{~mm}$. Measurements made on this sample using pulsed NMR at $4.7 \mathrm{~T}$ indicated $\mathrm{T}_{2}{ }^{*}$ and $\mathrm{T}_{1}$ values of approximately $50-60 \mathrm{~ms}$ and $310 \mathrm{~ms}$ respectively, in agreement with results reported elsewhere [32]. Figure 7 shows a photograph of the sample and the image that was acquired using the following parameters: 180 projections, 20 signal averages, a magnetic field gradient of $80 \mathrm{mT} / \mathrm{m}$, a magnetic field modulation of $80 \mu \mathrm{T}$, and an RF power of $7 \mathrm{dBm}$. The total acquisition time was 10 hours. Individual peanuts, which have a higher density of ${ }^{1} \mathrm{H}$ nuclei than the surrounding caramel, can be clearly discerned. Despite using an RF power half that used in previous experiment, some $\mathrm{T}_{1}$-induced saturation of the spin system did occur, manifest as a streaking artifact predominately towards the top of the image.

\section{Conclusions}


Continuous wave NMR imaging has been successfully applied to the study of various heterogeneous materials. Two-dimensional images were obtained of samples with $\mathrm{T}_{2}$ values ranging from approximately $50 \mathrm{~ms}$ down to $10 \mu \mathrm{s}$, demonstrating the versatility of the technique in spanning a broad range of $\mathrm{T}_{2}$ values. A spatial resolution of the order of $1 \mathrm{~mm}$ was realised for samples with $\mathrm{T}_{2}$ less than $40 \mu$ s when imaged using a gradient strength of $200 \mathrm{mT} / \mathrm{m}$. The prototype CW-MRI system was able to map inhomogeneities within cement plug samples, and revealed differences in the water penetration properties of two sandstone reservoir core plug samples deriving from their layering structure. The prototype system is currently being upgraded to enable 3-dimensional imaging at higher field gradients, as well as imaging of other nuclei such as ${ }^{31} \mathrm{P}$ to expand the range of applications of the technique.

\section{Acknowledgements}

This work was funded by the UK Engineering and Physical Sciences Research Council (Grant Number GR/R02269/01). The authors thank Dr. H. Seton, Department of Biomedical Physics, for help with the measurements on the $4.7 \mathrm{~T}$ system, and Dr. S. Ogilvie, Department of Geology, University of Aberdeen, for supplying the sandstone samples.

\section{References}

1. P. Blumler and B. Blumich, NMR Imaging of Solids, In: "NMR - Basic Principles and Progress", Eds.: P. Diehl, E. Flock, and R. Kosfeld, 30, 208-277, Springer, Berlin, Germany, (1994).

2. P. Jezzard, J. J. Attard, T. A. Carpenter, and L. D. Hall, Nuclear-magneticresonance imaging in the solid-state, Prog. Nuc. Mag. Res. Sp., 23, 1-41, (1991). 
3. D. E. Demco and B. Blumich, Solid-state NMR imaging methods. Part I: Strong field gradients, Concept Magnetic Res. , 12, 188-206, (2000).

4. E. R. Andrew and E. Szczesniak, A historical account of NMR in the solid state, Prog. Nuc. Mag. Res. Sp., 28, 11-36, (1995).

5. P. J. McDonald and B. Newling, Stray field magnetic resonance imaging, Rep. Prog. Phys., 61, 1441-1493, (1998).

6. S. L. Codd, M. J. D. Mallett, M. R. Halse, J. H. Strange, W. Vennart, and T. van Doom, A three-dimensional NMR imaging scheme utilizing doubly resonant gradient coils, J. Magn. Reson. B, 113, 214-221, (1996).

7. B. J. Balcom, R. P. MacGregor, S. D. Beyea, D. P. Green, R. L. Armstrong, and T. W. Bremner, Single-point ramped imaging with T-1 enhancement (SPRITE), J. Magn. Reson. A, 123, 131-134, (1996).

8. A. A. Samoilenko, D. Y. Artemov, and L. A. Sibeldina, Formation of sensitive layer in experiments of NMR subsurface imaging of solids, JETP Lett+, 47, 417-419, (1988).

9. S. P. Cottrell, M. R. Halse, and J. H. Strange, NMR imaging of solids using large oscillating field gradients, Meas. Sci. Techol., 1, 624-629, (1990).

10. Y. M. Daud and M. R. Halse, 2-dimensional Fourier transform NMR imaging of solids using large oscillating field gradients, Physica B, 176, 167-172, (1992).

11. S. Emid, Ultra high-resolution multiple-quantum spectroscopy in solids, Physica $B$ \& $C, \mathbf{1 2 8}, 79-80$, (1985).

12. I. V. Mastikhin, B. J. Balcom, P. J. Prado, and C. B. Kennedy, SPRITE MRI with prepared magnetization and centric k-space sampling, J. Magn. Reson., 136, 159-168, (1999).

13. F. D. Cano, T. W. Bremner, R. P. McGregor, and B. J. Balcom, Magnetic resonance imaging of $\mathrm{H}-1, \mathrm{Na}-23$, and $\mathrm{Cl}-35$ penetration in Portland cement mortar, Cement Concrete Res., 32, 1067-1070, (2002). 
14. A. J. Bohris, U. Goerke, P. J. McDonald, M. Mulheron, B. Newling, and B. Le Page, A broad line NMR and MRI study of water and water transport in Portland cement pastes, Magn. Reson. Imaging, 16, 455-461, (1998).

15. J. Tritt-Goc, N. Pislewski, A. Kaflak-Hachulska, D. Chmielewski, A. Gorecki, and W. Kolodziejski, Proton magnetic resonance microimaging of human trabecular bone, Solid State Nucl. Mag., 15, 91-98, (1999).

16. M. Peyron, G. K. Pierens, A. J. Lucas, L. D. Hall, and R. C. Stewart, The modified stretched-exponential model for characterization of NMR relaxation in porous media, J. Magn. Reson. A, 118, 214-220, (1996).

17. E. Laganas, G. Papavassiliou, M. Fardis, A. Leventis, F. Milia, E. Chaniotakis, and C. Meletiou, Analysis of complex H-1 nuclear-magnetic-resonance relaxation measurements in developing porous structures - a study in hydrating cement, J. Appl. Phys., 77, 3343-3348, (1995).

18. J. J. Attard, P. J. McDonald, S. P. Roberts, and T. Taylor, Solid-state NMR imaging of irreducible water in reservoir cores for spatially-resolved pore surface relaxation estimate, Magn. Reson. Imaging, 12, 355-359, (1994).

19. J. Godward, E. Ciampi, M. Cifelli, and P. J. McDonald, Multidimensional imaging using combined stray field and pulsed gradients, J. Magn. Reson., 155, 92-99, (2002).

20. A. Chowdhury, A. Gillies, P. J. McDonald, and M. Mulheron, Vapour phase application of hydrophobic coatings to cement- based materials, Mag. Concrete Res., 53, 347-352, (2001).

21. P. J. McDonald, The application of broad line MRI to the study of porous media, Magn. Reson. Imaging, 14, 807-810, (1996).

22. P. J. McDonald, Stray field magnetic resonance imaging, Prog. Nuc. Mag. Res. Sp., 30, 69-99, (1997). 
23. P. Szomolanyi, D. Goodyear, B. Balcom, and D. Matheson, SPIRAL-SPRITE: a rapid single point MRI technique for application to porous media, Magn. Reson. Imaging, 19, 423-428, (2001).

24. I. V. Mastikhin, H. Mullally, B. MacMillan, and B. Balcom, Water content profiles with a 1D centric SPRITE acquisition, J. Magn. Reson., 156, 122-130, (2002).

25. S. D. Beyea, B. J. Balcom, I. V. Mastikhin, T. W. Bremner, R. L. Armstrong, and P. E. Grattan-Bellew, Imaging of heterogeneous materials with a turbo spin echo single-point imaging technique, J. Magn. Reson., 144, 255-265, (2000).

26. G. R. Davies, D. J. Lurie, J. M. S. Hutchison, S. J. McCallum, and I. Nicholson, Continuous-wave magnetic resonance imaging of short T-2 materials, J. Magn. Reson., 148, 289-297, (2001).

27. C. B. Kennedy, B. J. Balcom, and I. V. Mastikhin, Three-dimensional magnetic resonance imaging of rigid polymeric materials using single-point ramped imaging with T-1 enhancement (SPRITE), Can. J. Chem., 76, 1753-1765, (1998).

28. J. J. Howard, Quantitative estimates of porous media wettability from proton NMR measurements, Magn. Reson. Imaging, 16, 529-533, (1998).

29. D. N. Guilfoyle, B. Issa, and P. Mansfield, Rapid volumetric NMR imaging of fluids in porous solids using a 3D pi-EPI (PEPI) hybrid, J. Magn. Reson. A, 119, 151-156, (1996).

30. M. Bencsik and C. Ramanathan, Direct measurement of porous media local hydrodynamical permeability using gas MRI, Magn. Reson. Imaging, 19, 379383, (2001).

31. M. K. Gingras, B. MacMillan, B. J. Balcom, T. Saunders, and S. G. Pemberton, Using magnetic resonance imaging and petrographic techniques to understand the textural attributes and porosity distribution in Macaronichnus-burrowed sandstone, J. Sediment. Res., 72, 552-558, (2002). 
32. M. E. Miquel and L. D. Hall, A general survey of chocolate confectionery by magnetic resonance imaging, Lebensm. -Wiss. u. -Technol., 31, 93-99, (1998). 


\section{Figure Legends}

Figure 1: Block diagram of the CW-MRI system. The dashed box outlines the elements of the basic CW-MR spectrometer.

Figure 2: (a) Schematic diagram, (b) unprocessed image and (c) deconvolved image of a poly(methyl methacrylate) (PMMA) resolution phantom $\left(T_{2}=38 \mu \mathrm{s}\right)$. Image acquisition time $=30$ minutes.

Figure 3: 1-dimensional profiling of a 0.4 water/cement sample for different curing and drying times. A gradient of $140 \mathrm{mT} / \mathrm{m}$ was used. The unsealed face was on the left side.

Figure 4: Measured linewidth of the 0.4 water/cement sample as a function of curing and drying times.

Figure 5: Water penetration into a cement sample following soaking times of:

(a) 5 minutes, (b) 10 minutes, (c) 15 minutes, (d) 30 minutes, (e) 60 minutes and (f) 5 hours. Image acquisition times $=15$ minutes.

Figure 6: Water penetration into the (a) 'parallel' and (b) 'perpendicular' sandstone reservoir core plug samples. The left sides were soaked between profiling experiments.

Figure 7: (a) Photograph and (b) CW-MRI image of a sample from a Snickers ${ }^{T M}$ confectionery bar measuring $42 \times 25 \times 4 \mathrm{~mm}$. Image acquisition time $=10$ hours. 
Figure 1

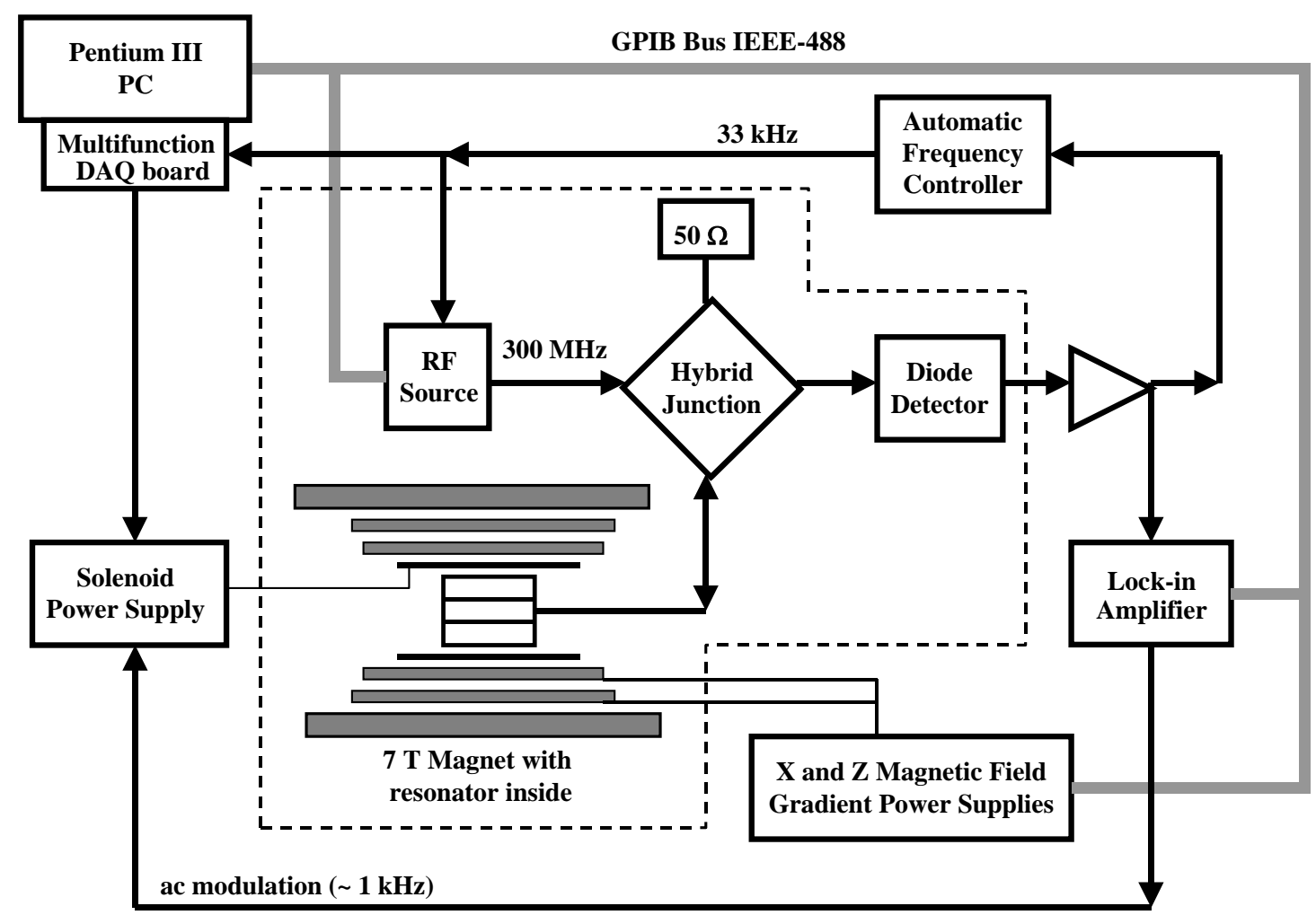


Figure 2
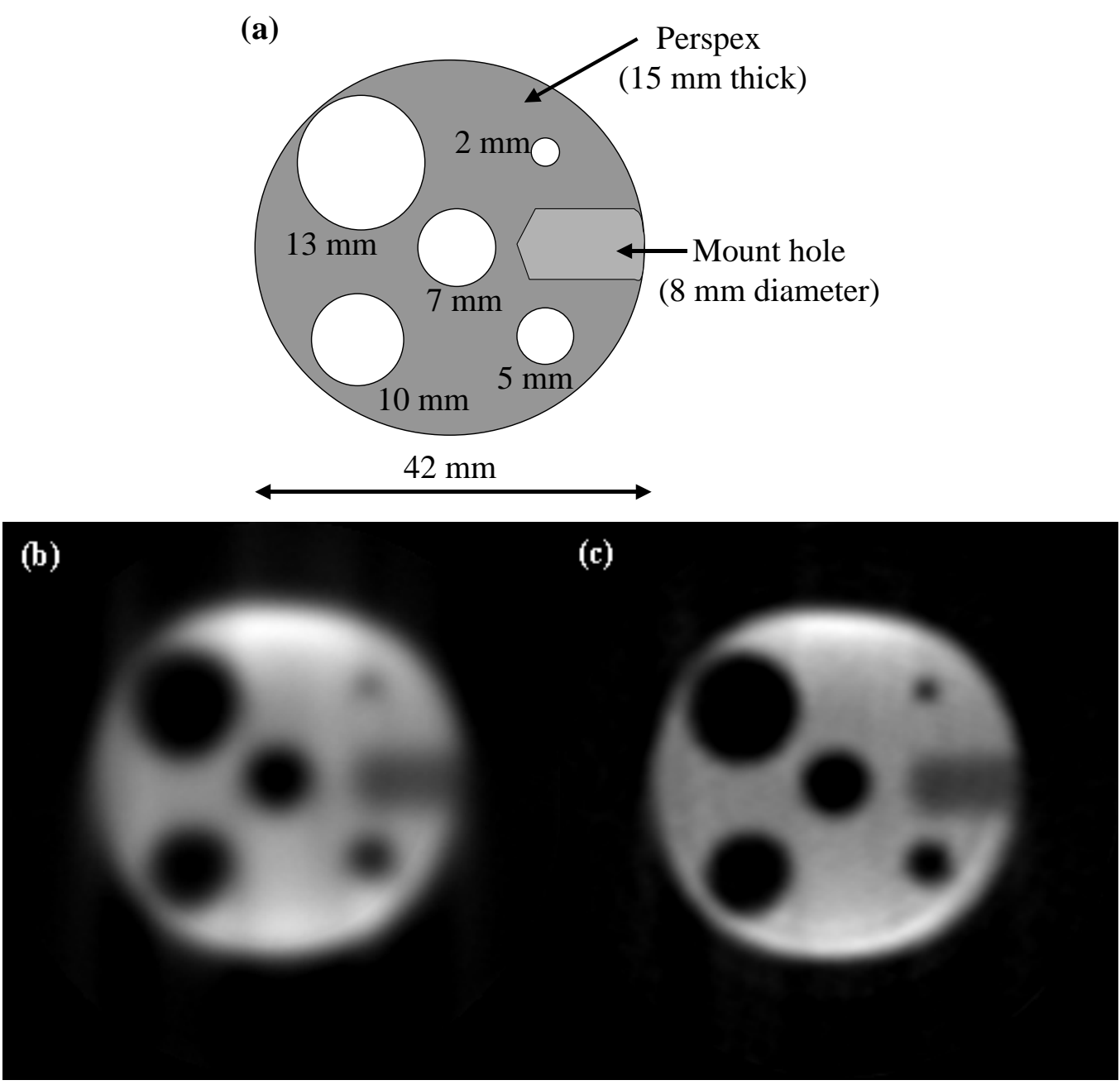
Figure 3

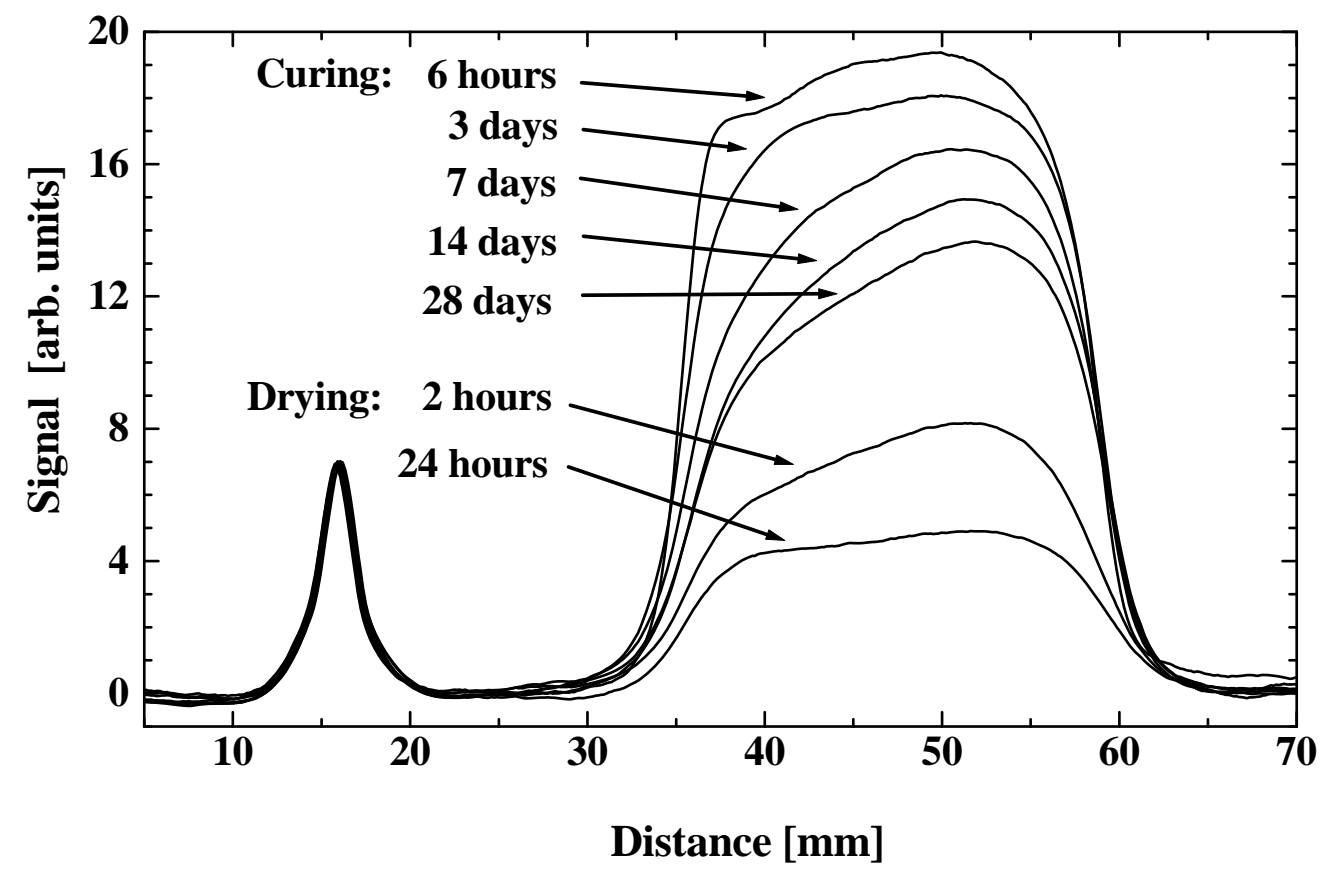


Figure 4

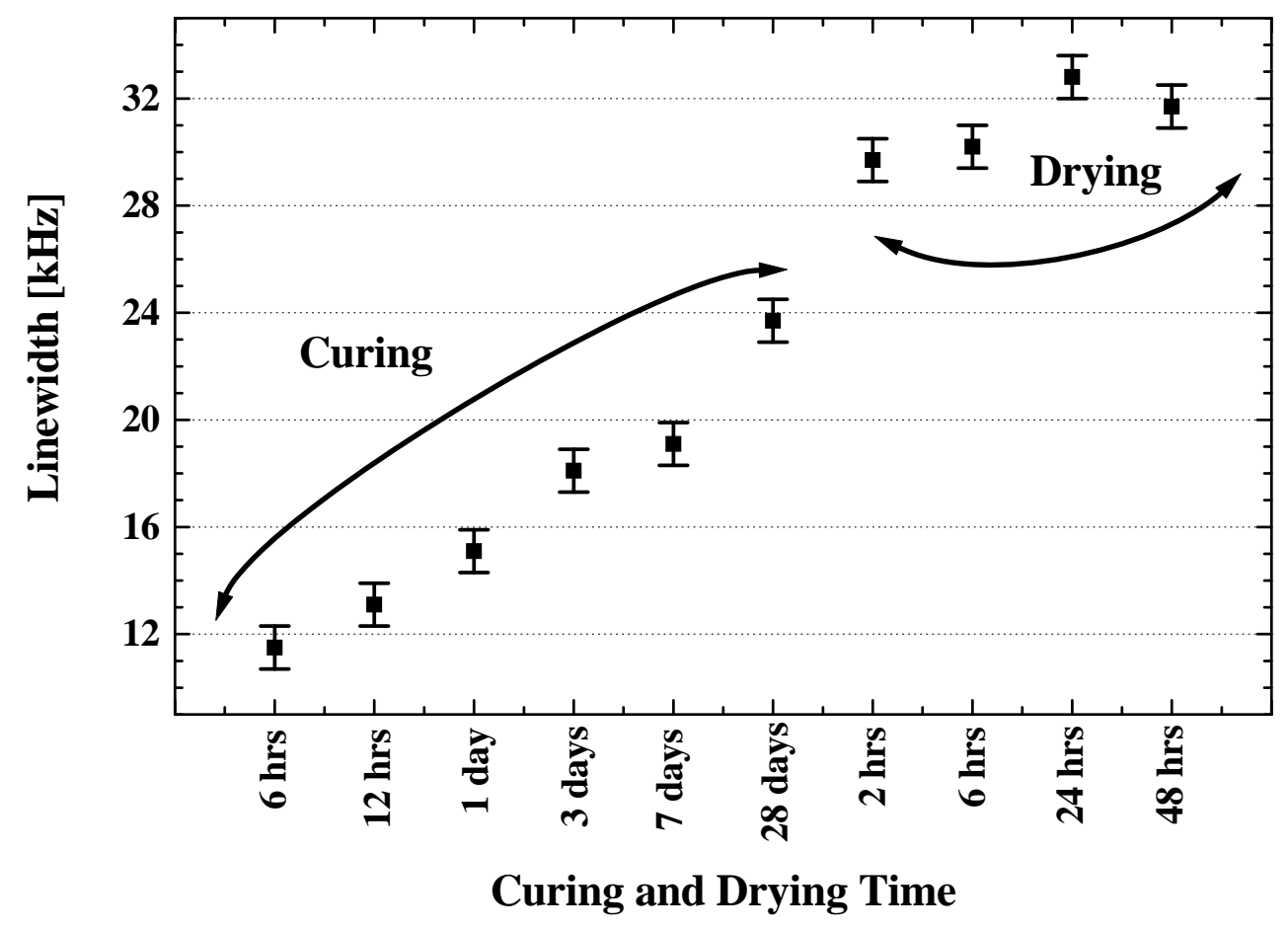


Figure 5

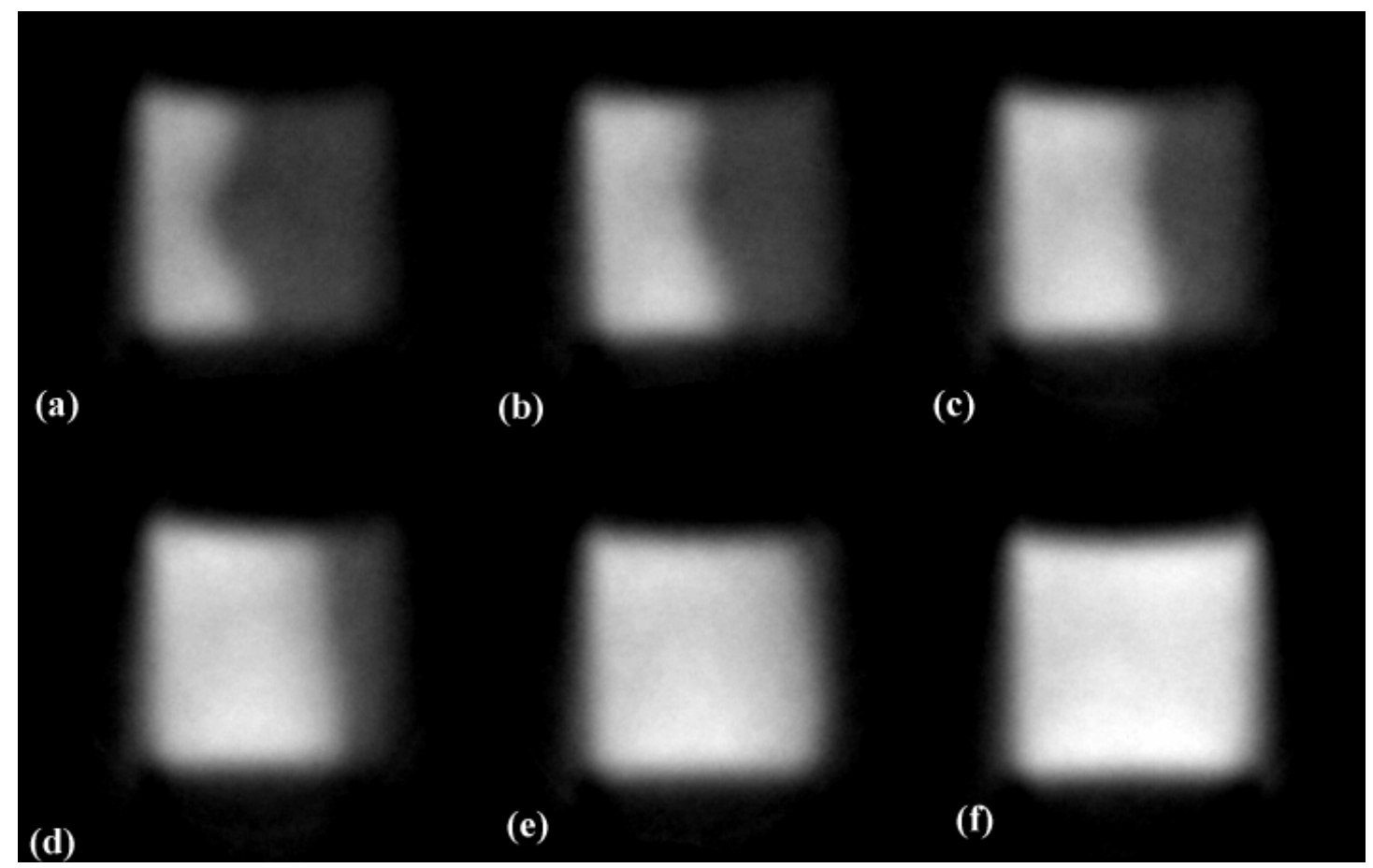

A.J. Fagan et al., Continuous Wave MRI of Heterogeneous Materials, page 22 of 24 
Figure 6

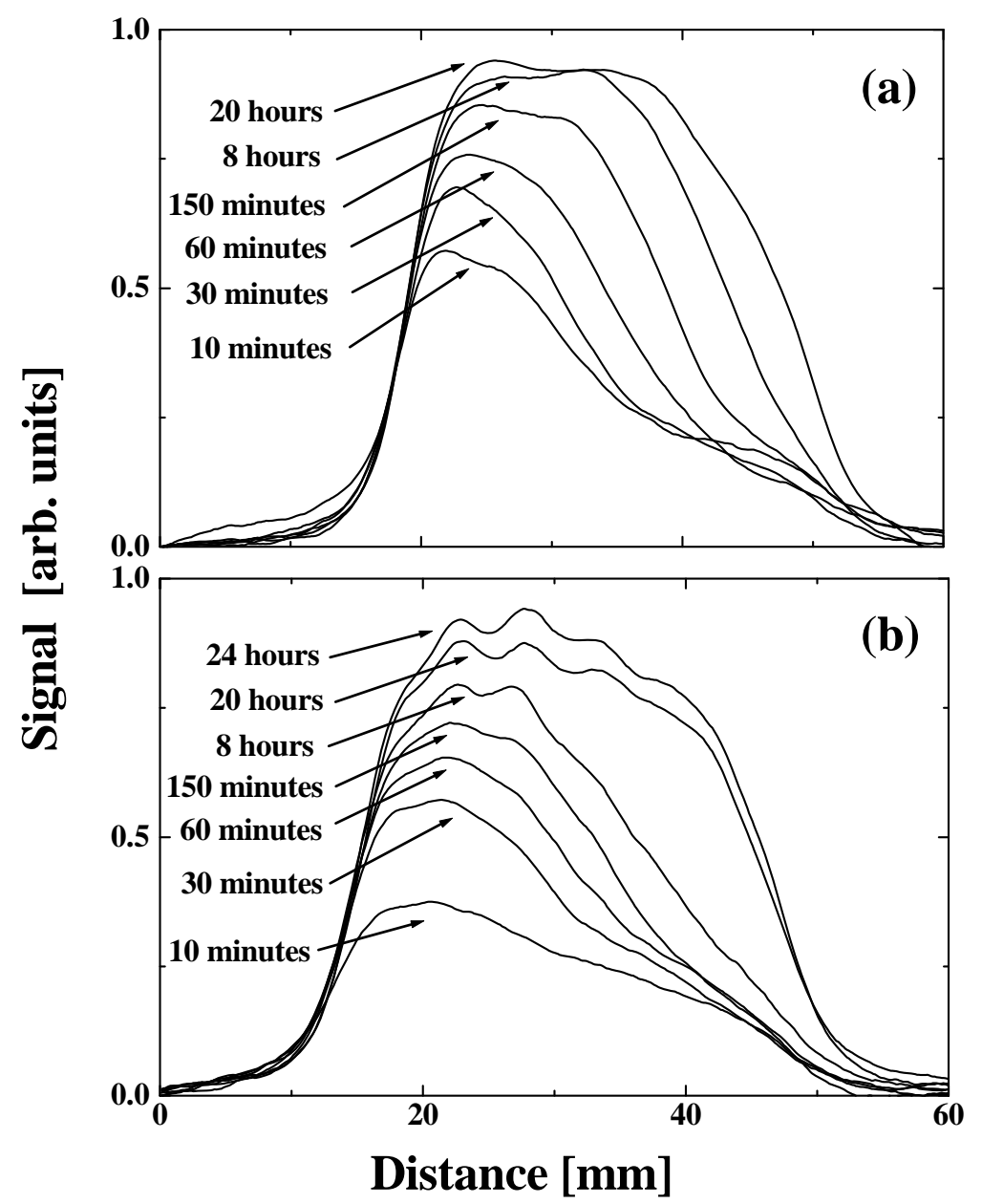


Figure 7

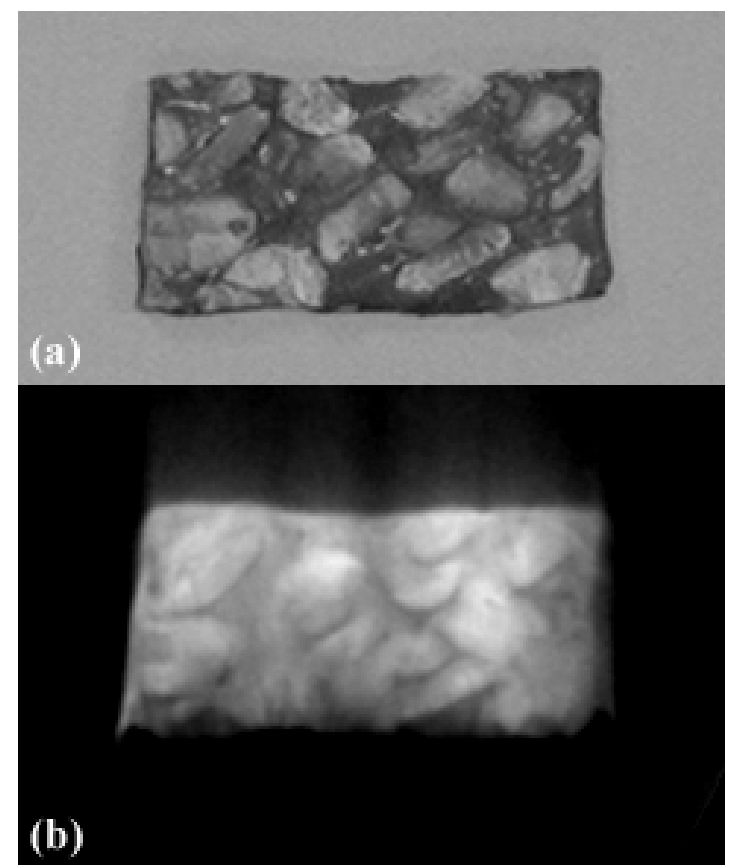

A.J. Fagan et al., Continuous Wave MRI of Heterogeneous Materials, page 24 of 24 